\title{
Evaluation de la dégradation du bois de conifères immergés durant plus d'un demi-siècle dans un réservoir ${ }^{1}$
}

\author{
R. Van Coillie 2 \\ S. A. Visser ${ }^{3}$ \\ P. G. C. Campbell \\ H. G. Jones
}

\begin{abstract}
Après 55 ans d'immersion continue dans un réservoir en forêt boréale, les troncs de conifères (Abies balsamea, Picea mariana et Pinus banksiana) sont peu modifiés. En effet, leur bois ne présente qu'unc faible dégradation périphérique. Leur pourcentage en matériel soluble dans $\mathrm{NaOH}(1 \%)$ et leur pourcentage en pentosans ont quelque peu diminué ; par contre, leur teneur en lignine acido-insoluble et leur densité de bois n'ont guère changé, ce qui montre que leur réseau ligneux dense reste quasiment inaltéré. Au niveau histologique, celui-ci présente plusieurs hyphes de champignons et des ponctuations aérolées agrandies. Ces faibles modifications résultent vraisemblablement d'une action microbiologique synergique des champignons de la carie molle et de certaines bactéries. Les conditions physicu-chimiques du réservoir ne s'avèrent pas incompatibles pour les décomposeurs bien que les basses températures qui y règnent favorisent peu leur action.
\end{abstract}

\section{A study of the breakdown of wood of conifers submerged for over half a century in a reservoir.}

After 55 years of continual submersion in a subarctic reservoir, the trunks of conifers (Abies balsamea, Picea mariana, and Pinus banksiana) are lit te modified and only slight peripheral degradation is visible. The content of $\mathrm{NaOH}(1 \%)$ extractible material and pentosans is slightly decreased, but changes in the level of acid-insoluble lignin and wood density are negligible, indicating that the dense lignous network remains practically unaltered. At the histulogical level, the woody tissue contains several fungal hyphae and exhibits enlarged bordered pits. These slight modifications presumably result from an additive microbiological action of soft-rot fungi and of certain bacteria. The physico-chemical conditions prevailing in the reservoir permit the development of these decomposers but the relatively low water temperature limits their action.

\section{1. - Introduction}

Pour répondre aux besoins de ressources énergétiques additionnelles, on aménage, entre autres, des réservoirs hydroélectriques à des altitudes et latitudes élevées où prédominent des conifères (Van Coillie et al. 1983). Une question se pose à ce sujet : faut-il déboiser les superficies destinées à être inondées ou les submerger telles quelles? La réponse dépend non seulement d'un facteur économique (coût de déboisement et de transport par rapport au

\footnotetext{
1. Etude réalisée à l'INRS-E:lu, Universit é du Quëbec. C.P. 7500. Sainte-For, Quebec GiV 4C7 CANADA.

2. Adresse actuelle: Conseil de la Science et la Technologie du Gouvemement du Québec. 1275 boulevard Charest Ouest, Québec, G1N 2 C 9 CANADA.

3. Adresse actuelle: Département des sols, Université Laval, Sainte.Foy, Québec G1K 7P4, CANADA.
}

revenu de vente) mais aussi d'un facteur environnemental, à savoir l'effet de la décomposition du bois sur la qualité du milieu aquatique. De ce fait, la décomposition végétale, en plus d'y réduire l'oxy. gène disponible (celui-ci étant pris par l'activité microbiologique de dégradation), peut libérer divers composés organiques dans un réservoir ; le phénomène, tout en étant moins prononcé que dans les régions chaudes (Leentvaar 1966), exerce une influence indéniable dans les régions froides (Rhode $1964 \mathrm{a}$ et b). On a notamment constaté dans ces dernières que, lors de l'aménagement de réservoirs, des composés organiques s'y retrouvent après quelques mois en quantité suffisante pour changer le potentiel de fertilité des eaux pour les algues (Visser et al. 1977 ; Van Coillie et al. 1981). Qu'en est-il à plus long terme? Afin de répondre à cette question, nous avons examiné la dégradation du bois de conifères 
après 55 ans d'immersion dans un réservoir en forêt boréale pour évaluer ce qu'elle a libéré dans celuici durant cette période.

\section{2. - Lleu d'échantillonnage}

Le réservoir hydroélectrique Gouin a été formé en 1916-1918 à la suite de la construction d'un barrage à la partie supérieure du bassin de la rivière SaintMaurice : son remplissage a duré 639 jours, soit 1,74 année. Ce réservoir est localisé à approximativement 320 kilomètres au nord de Montréal entre les latitudes $48^{\circ} 15^{\prime} \mathrm{N}$ et $48^{\circ} 45^{\prime} \mathrm{N}$ et les longitudes $74^{\circ} 05^{\prime}$ et $74^{\circ} 50^{\prime}$ (fig. 1). Il est un des plus vicux réservoirs hydroélectriques du Québec, couvre $960 \mathrm{~km}^{2}$ et renferme les vestiges de la végétation qui y existait avant l'inondation. Ses fluctuations annuelles de niveau se situent entre les cotes 394 et $405 \mathrm{~m}$ avec une profondeur moyenne d'environ $10 \mathrm{~m}$. La qua. lité de l'eau du réservoir a été étudiée durant 2 étés (1973, 1974) par une équipe d'Hydro-Québec ; à titre d'exemple, on présente dans le tableau I les concentrations moyennes obtenues pour divers parametres

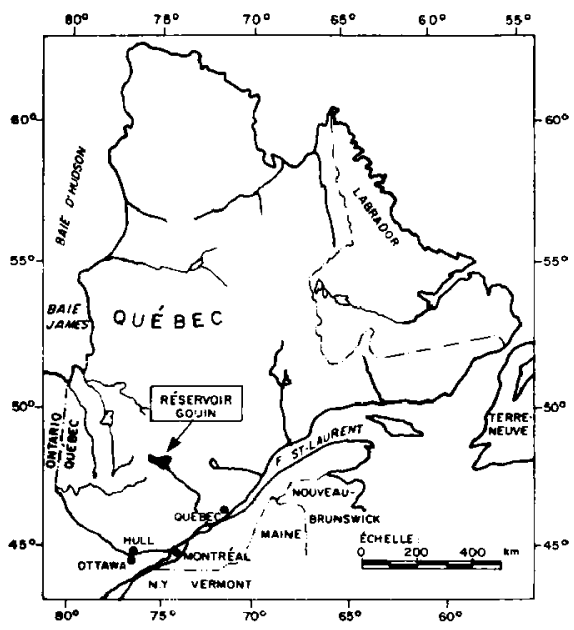

Fig. 1. Localisation géographique du réservoir Gouin. à 19 stations dans le réservoir. La qualité globale de l'eau du réservoir correspond aux caux faiblement minéralisées que l'on trouve habituellement sur le Bouclier canadien au Québec (Bobée et al. 1977). Lors de la prise des échantillons de bois dans l'eau, en octobre 1972, la température aquatique y équivalait à $4.5^{\circ} \mathrm{C}$, le $\mathrm{pH}$ à 6,25 , la conductivité à $65 \mu \mathrm{S} / \mathrm{cm}$ et l'oxygène dissous à $11,1 \mathrm{mg} / \mathrm{en}$ surface.

Tableau I. Valeurs moyennes de quelques paramètres de qualite de l'eau du réservoir Gouin'1.

1. G. Guertin, Hydro-Québec, communication personnelle

2. Concentrations exprimées en mg/l, sauf pour le $\mathrm{pH}$.

\begin{tabular}{|c|c|c|}
\hline PARAMETRE & & CONCENTRATION \\
\hline $\begin{array}{l}\text { sodium } \\
\text { potassium } \\
\text { fer } \\
\text { dureté }\end{array}$ & $\left(\mathrm{CaCO}_{3}\right)$ & $\begin{array}{l}0,55 \\
0,35 \\
0,06 \\
8,0\end{array}$ \\
\hline $\begin{array}{l}\text { chlorures } \\
\text { nitrates } \\
\text { phosphates totaux }\end{array}$ & $\begin{array}{c}\left(\mathrm{N}-\mathrm{NO}_{3}\right) \\
\left(\mathrm{P}-\mathrm{PO}_{4}\right)\end{array}$ & $\begin{array}{l}5,5 \\
0,05 \\
0,02\end{array}$ \\
\hline tannins & & 0,93 \\
\hline $\begin{array}{l}\text { solides en suspension } \\
\text { pH } \\
\text { oxygene dissous }\end{array}$ & & $\begin{array}{r}6,0 \\
6,2 \\
11,2\end{array}$ \\
\hline
\end{tabular}

\section{3. - Matériel et méthodes}

Des plongeurs équipés de phares (vu la couleur foncée de l'eau) scièrent transversalement des sections de troncs de conifères suivants à $1,6 \mathrm{~km}$ en amont du barrage du réservoir et à $0,5 \mathrm{~km}$ de son rivage sud : Abies balsamea (sapin baumier) et Picea mariana (épinette noire), constamment submergés depuis l'inondation de ce site dans le réservoir au cours de l'été 1917 et extraits d'une profondeur de $10,7 \mathrm{~m}$ (à $0,3 \mathrm{~m}$ au-dessus du fond). Les sections prises avaient une hauteur de $19 \mathrm{~cm}$ et un diamètre de 8 à $13 \mathrm{~cm}$.

Pres du mème site, on découpa également des sections de grandeur analogue dans des troncs de Pinus banksiana (pin gris) qui, depuis l'été 1917, avaient été inondés de façon intermittente selon le niveau d'eau du réservoir ; au moment de l'échantillonnage, ce bois se prolongeait à $0,7 \mathrm{~m}$ au- dessus de la surface de l'eau.

Immédiatement après leur prisc, les spécimens de bois du fond du réservoir furent placés dans des sacs 
de plastique remplis d'eau provenant de l'endroit où le bois avait été recueilli. Par contre, les pièces de bois immergées de façon intermittente étaient déposées dans des sacs de plastique secs. Ensuite, les échantillons et leur contenu furent conservés au sein d'une boite réfrigérée à $0-4^{\circ} \mathrm{C}$ jusqu'à Québec où ils furent entreposés dans une chambre froide à $4^{\circ}$ $C$ en vue de leur analyse ultérieure.

Pour permettre l'identification des espèces d'arbres submergés (de façon constante ou intermittente) à l'aide d'examens morphologiques comparatifs et pour disposer de témoins, on scia des sections transversales de troncs des différentes espèces majoritaires d'arbres présentes au bord du réservoir. Ces échantillons furent ensuite conservés de la même façon que les autres spécimens issus des troncs immergés avec intermittence.

Des portions des différents spécimens ont été analysées pour leur densité et leurs pourcentages en lignine acido-insoluble, en matériel soluble dans $\mathrm{NaOH}(1 \%)$ et en pentosans, selon des méthodes couramment utilisées à cette fin dans l'industrie des pâtes et papiers pour évaluer le degré de dégrada. tion du bois (Tappi 1970).

Dans des petits cubes des portions périphérique, centrale et intermédiaire des spécimens d'Abies balsamea et de Picea mariana, on effectua des coupes de $25 \mu \mathrm{m}$ d'épaisseur selon les trois plans de symétrie du bois, à savoir transversal, radial et tangentiel. Ces coupes furent ensuite colorées au violet de méthyl et au brun de Bismarck (Gabe 1968) afin d'y distinguer respectivement les structures du bois et les hyphes de champignons.

\section{4. - Résultats et discussion}

Après 55 ans d'immersion continue ou intermittente, les troncs des trois espèces étudićes présentaient une écorce irrégulièrement détachée. Hormis cette particularité, leurs sections transversales respectives de bois avaient presque le même aspect que celles des troncs des trois espèces au bord du réservoir, ce qui facilita leur identification spécifique. Nonobstant cette ressemblance, on vérifia si des différences étaient survenues aux niveaux de la densité, de la composition chimique et de la texture histologique entre les bois qui furent submergés et ceux qui ne le furent point.

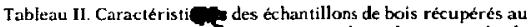
réservoir Gouin et comparaison avec des valeurs standards pour des echantillons nayant jamais été submergés.

1. Les valeurs standards correspondent a celles définies par le Centre canadien de Recherches en Pâtes et Papiers (Berzins, 1966).

2. La lignine acido-soluble ne dépassait guere 0.3 à $0,4 \%$.

\begin{tabular}{|c|c|c|c|c|}
\hline $\begin{array}{l}\text { Espece } \\
\text { de } \\
\text { conifere }\end{array}$ & $\begin{array}{l}\text { Densité } \\
\left(\mathrm{g} / \mathrm{cm}^{3}\right)\end{array}$ & $\begin{array}{l}\text { Lignine } \\
\text { acido- } \\
\text { insolubie }\end{array}$ & $\begin{array}{l}\text { Materie] } \\
\text { soluble } \\
\text { dans } \\
\mathrm{NaOH} \text { (1\%) }\end{array}$ & Pentosans \\
\hline $\begin{array}{l}\text { Abies balsamca } \\
\text { standard' } \\
\text { submergé } \\
\text { (continuellement) }\end{array}$ & $\begin{array}{l}0.34 \\
0,33\end{array}$ & $\begin{array}{l}27,9 \\
30,3\end{array}$ & $\begin{array}{l}12,5 \\
11,5\end{array}$ & $\begin{array}{l}9.3 \\
7.5\end{array}$ \\
\hline $\begin{array}{l}\text { Picea mariana } \\
\text { standard! } \\
\text { submergé } \\
\text { (continuellement) }\end{array}$ & $\begin{array}{l}0,38 \\
0,39\end{array}$ & $\begin{array}{l}27,3 \\
29,1\end{array}$ & $\begin{array}{l}14,1 \\
11,0\end{array}$ & $\begin{array}{l}9,6 \\
8,7\end{array}$ \\
\hline $\begin{array}{l}\text { Pinus banksiana } \\
\text { standard } \\
\text { submergé } \\
\text { (discontinuel- } \\
\text { lement) }\end{array}$ & $\begin{array}{l}0,39 \\
0,38\end{array}$ & $\begin{array}{l}27,0 \\
30,1\end{array}$ & $\begin{array}{l}12,7 \\
12,3\end{array}$ & $\begin{array}{r}10.5 \\
9.0\end{array}$ \\
\hline
\end{tabular}

Les analyses des densités et des principaux critères biochimiques donnèrent les résultats présentés dans le tableau II. Signalons que le taux de matériel soluble dans $\mathrm{NaOH}(1 \%)$ est un paramètre qui évalue la qualité du bois comme matière primaire pour la fabrication du papier. A ce titre, il constitue un indice de la dégradation du bois; sa valeur normale se situe à environ $14 \%$ et, au delà de $20 \%$, il traduit une dépolymérisation des celluloses et hémicelluloses. Quant à la teneur en pentosans, elle reflète le degré d'utilisation microbiologique des hémicelluloses. Les polymères de pentoses sont presqu'exclusivement associés aux hémicelluloses des fibres. Une dégradation accrue de la matière cellulosique, indiquée par le test de dissolution dans $\mathrm{NaOH}(1 \%)$, devrait donc normalement se traduire par une diminution des pentosans, pour autant que ces derniers aient été utilisés par les microorganismes de décomposition.

A l'examen de l'ensemble des résultats (Tableau II), il appert qu'un séjour continu ou discontinu du bois de coniferes dans un réservoir nordique durant plus d'un demi-siècle n'affecte que peu son matériel soluble dans $\mathrm{NaOH}(1 \%)$ et ses pentosans. De plus, sa lignine ne semble guère altérée : le fait que la densité de ce bois, laquelle dépend surtout de l'intégrité du réseau ligneux (Sarkanen et Ludwig 1971), n'ait pas changé significativement, soutient indirectement cette conclusion. 


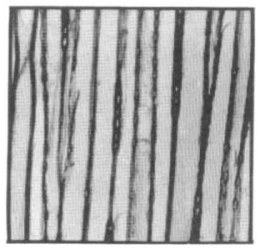

Figure 2.1

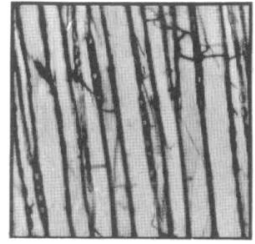

Figure 2.2

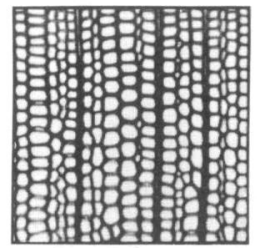

Figure 2.3

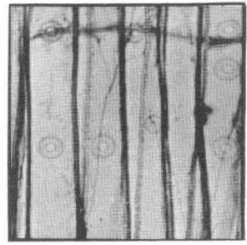

Figure 2.4

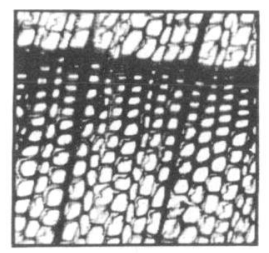

Figure 2.5

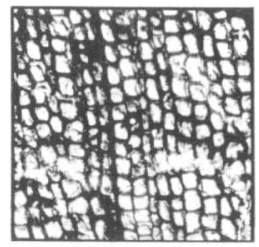

Figure 2.6

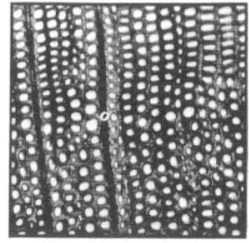

Figure 2.7

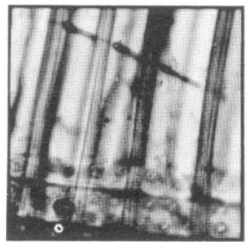

Figure 2.8

Fig. 2. Caractéristiques histologiques du bois de conifères en conditions normales et après longue immersion.

Fig. 2.1. A bies balsamea jamais submergé (témoin) ; coupe tangentielle dans la région périphérique. On y aperçoit une juxtaposition de trachéides verticales $(100 \mathrm{x})$.

Fig. 2.2. Albies balsamea submergé durant 55 ans ; coupe tangentielle darts la région périphérique. On constate une légère altération des trachéides axiales (100 x).

Fig. 2.3. Abies balsamea submergé durant 55 ans; coupe transversale dans la région intermédiaire. Les trachéides du a bois d'été " (étroites) et du "bois de printemps" (larges) présentent un aspect normal (100 x).

Fig. 2.4. Abies balsamea submergé durant 55 ans; coupe radiale dans la région périphérique. Les trachéides longitudinales sont transpercées à certains endroits par des hyphes et leurs ponctuations aérolées ont un orifice central agrandi $(250 \mathrm{x})$.

Fig. 2.5. Picea mariana jamais submergé (témoin); coupe transversale dans la région périphérique. On y distingue une succession de trachéides étroites d'été et de trachéides larges de printemps. Perpendiculairement à cette succession, on remarque un rayon ligneux $(100 \mathrm{x})$.

Fig. 2.6. Picea mariana submergé durant 55 ans; coupe transversale dans la région périphérique. Par rapport à la figure 2.5, il appert qu'une dégradation morphologique diffuse est survenue (100 $\mathrm{x}$ ).

Fig. 2.7. Picea mariana submergé durant 55 ans ; coupe transversale dans la région intermédiaire. Les trachéides du " bois d'été " et du a bois de printemps "et les rayons ligneux ont conservé un aspect normal (100 x).

Fig. 2.8 Picea mariana submergé durant 55 ans; coupe radiale dans la région périphérique. Les trachéides longitudinales sont transpercées à certains end roits par des hyphes au niveau des rayons ligneux tranșversaux (partie inférieure) et en dehors de ceux-ci (partie supérieure). Nombre de ponctuations aérolées apparaissent percées à la hauteur des associations entre trachéides et rayons ligneux $(400 \mathrm{x}$ ). 
Au point de vue histologique, on constate que le bois d'Abies balsamea et de Picea mariana ayant séjourné 55 ans au fond du réservoir montre une dégradation périphérique partielle et limitée par rapport à l'aspect structural qu'il présente en périphérie dans les troncs témoins de ces espèces (fig. 2.1 et 2.2 ; fig. 2.5 et 2.6 ). Par contre, aucune dégradation n'est perceptible dans les régions intermédiaires et centrales du bois de ces deux espèces après 55 ans d'immersion ( $f$ ig. 2.3 et 2.7). Au sein des zones périphériques quelque peu altérées, on peut détecter des hyphes perforateurs de champignons tant au niveau des trachéides longitudinales qu'au niveau des rayons ligneux transversaux associés à celles-ci ( $f$ ig. 2.4 et 2.8 ). En outre, les ponctuations aérolées dans les régions périphériques apparaissent élargies ou transpercées, vraisemblablement par des bactéries. Ces deux dernières particularités s'avèrent rares dans les régions intermédiaires et centrales du bois submergé.

Les résultats chimiques et histologiques montrent donc que les changements survenant dans le bois de conifères durant 55 ans d'immersion en conditions nordiques semblent mineurs, excepté au niveau de leur écorce qui se détache. Les changements sont surtout d'ordre microbiologique, comme le révèlent certaines observations histologiques (hyphes de champignons et ponctuations aérolées agrandies par une intervention bactérienne). De fait, les agents microbiologiques qui peuvent s'adapter aux conditions physico-chimiques du fond d'un réservoir nordique sont principalement des ascomycètes de la carie molle et des bactéries (Levy 1965, Greaves 1971).

La carie molle se caractérise par la formation d'hyphes (cavités allongées et ramifiées dans la couche centrale de la paroi secondaire des trachéides et des rayons ligneux) dans les derniers cernes annuels de l'aubier et à la région périphérique du bois (Levy 1965). Elle dégrade surtout la cellulose, principalement celle qui a un poids moleculaire relativement faible (Levi et Preston 1965). En ce qui concerne les hémicelluloses et les pentosans, on constate qu'elles sont peu at taquées. Il ne semble pas y avoir d'accumulation de produits primaires de dégradation au-delà des concentrations immédiatement utilisables par les champignons (Levi et Preston 1965). Quant à la lignine, elle subirait une déméthylation progressive de ses radicaux méthoxy $\left(-\mathrm{OCH}_{3} \rightarrow-\mathrm{OH}\right.$ ) lors de l'action des champignons de la carie molle, mais ceci $n$ 'affecterait quasiment pas le réseau macromoléculaire ligneux (Kirk 1971). Signalons ici que la carie molle se présente moins souvent dans le bois des conifères que dans celui des feuillus. Cette résistance a été at tribuće à la teneur élevée en lignine et à l'imperméabilité relative du bois des gymnospermes comparativement à celles des angiospermes (Levy 1965).

Après l'attaque initiale du bois par les champignons de la carie molle, les bactéries commencent à dégrader les parois cellulaires en se servant des ponctuations existantes. Cette action accroit beaucoup la perméabilité du bois, lequel effet s'avère net après des séjour's du bois dans l'eau durant quelques années (Adolf et al. 1972). La poussée bactérienne se réalise principalement au niveau des polysaccharides structuraux; parmi ceux-ci, la cellulose est beaucoup plus affectée que les hémicelluloses et les pentosans (Greaves 1971), similairement à ce qu'on constate pour la carie molle (voir plus haut) avec toutefois une accumulation plus prononcée de produits primaires de dégradation. De même que lors de l'intervention de la carie molle, la lignine, pour sa part, résiste à l'action bactérienne, tout en présentant une déméthylation importante de ses groupements méthoxy (Kirk 1971). Etant donné que, dans le bois de coeur, les tores lignifiés ne favorisent guère l'envahissement et ensuite la multiplication des bactéries dans cette partie du bois (Fogarty 1973), la dégradation bactérienne se limite aux derniers anneaux annuels situés à l'extérieur de l'aubier, comme c'est le cas pour la carie molle.

Vu que le bois a une résistance due à sa nature chimique polymère prédominante et à l'insolubilité de ses constituants majeurs dans l'eau, la biodégradation s'effectue en général de façon très lente en milieu aquatique. Le fait que les unités monomères de la lignine s'avèrent variées, de même que ses liaisons intermonomères, entraîne une stabilité intramoléculaire très élevée et, aussi longtemps que la texture du bois reste intacte, les constituants glucidiques secondaires tels que les hémicelluloses et les pentosans seront peu disponibles pour la biodégradation.

Dans le cas présent, l'action microbiologique sur le bois de conifères immergés durant 55 ans dans le réservoir se révèle indéniablement faible. Ceci s'explique si l'on tient compte du fait que, malgré le synergisme d'action qui existe probablement entre les champignons et les bactéries, les basses 
températures du réservoir ont sans doute beaucoup limité l'action microbiologique. Par ailleurs, les quelques changements chimiques constatés se décèlent non seulement dans la partie externe du bois des conifères mais aussi, à plus faible degré, dans ses parties intérieures. Ceci pourrait être le résultat de la sécrétion par les bactéries et les champignons d'ectoenzymes hydrolysantes qui sont diffusées avec l'eau dans le bois (Levy 1965, Fogarty et Ward 1972).

L'ensemble des données précédentes fait donc ressortir que la biodégradation du bois de conifères dans le milieu aquatique s'arère "lente mais inévitable s sous des conditions nordiques.

\section{Remerclements}

Cette recherche a été demandée par Hydro-Quebec. Les auteurs tiennent à exprimer leur gratitude au docteur M. Meybeck (stagiaire à l'INRS-Eau, Université du Québec, en 1972-73), au professeur P. Poliquin (Faculté de foresterie, Université Laval), à I'Institut canadien de Recherches en Pátes et Papiers et à Messieurs R. Bérubé, G. Guertin et L. P. Roy de la direction d'Environnement d'Hydro-Québec pour leurs contributions à l'étude.

\section{Travaux cités}

Adolf (B.), Gerstetter (E.) et Liese (W.). 1972. - * Untersuchungen über einige Eigenschaften von Fichtenholz nach dreijahriger Wasserlagerung *. Holzforschung, 26: 18-25.

Bobée (B.), Cluis (D.), Goulet (M.), Lachance (M.), Potvin (L.) et Tessier (A.). 1977. - Evaluation du réseau de la qualité des eaux (Ministère des Richesses naturelles du Québec) : analyse et interprétation des données de la période 1967.75 ». Volume 1 (texte, ableaux et figures) ; volume 2 (annexes). INRS.Eau, Université du Québec, Rapport scientifique $n^{\circ} 78,278$ p.

Berzins (V.). 1966. - . Chemical composition of woods *. Pulp and Paper Research Institute of Canada, Pointe Claire, Québec. Reseatch Note $n^{\circ} 61,9 p$.
Fogarty (W.M.). 1973. - * Bacteria, enzymes and wood permea. bility *. Process Biochem., $8: 30-34$

Fogarty (W.M.) Ward (O.P.). 1972, — "Enzyme production by bacteria isolated from water-stored Sitka Spruce (Picea sitchensis) o. J. appl. Bact., 35: 685-689.

Gabe (M.). 1968. - *Techniques histologiques *. Editions Masson, Paris, 1113 p.

Greaves (H.). 1971. - The bacterial factor in wood decay * Wood Sci. Technol., 5: 6-16.

Kirk (I.K.). 1971. - Effects of microorganisms on lignin * Ann. Rev. Phytopath., 9:185-210.

Leentvaar (P.). 1966. - "The Brokopondo lake in Surinam *. Verh. Intem. Verein. Limnol., 16: 680-684.

Levi (M.P.) Preston (R.D.). 1965. - A chemical and microscopic examination of the action of the soft-rot fungus Chaetomium glabosum on beechwood (Fagus syly) *. Holzforschung, 19: 183.190.

Lev (J.). 1965. - The soft-rot fungi : their mode of action and significance in the degradation of wood. = In : * Advances in Botanical Research *. R. D. Preston (ed.). Academic Press, N. Y., 2: 323-357.

Rhode (W.). 1964a, - "Effects of impoundment on water che mistry and plankton in Lake Ransaran ". Verh. Intern. Verein. Limnol, $15: 437.443$.

Rhode (W.). 1964b. - "Effects of impoundment on the feeding habits of brown trout and char in Lake Ransaran ". Verh. Intem. Verein. Limnol., is : 444.452.

Sarkanen (K.V.) Ludwig (C.H.). 1971. - * Lignins: occurrence, formation, structure and reaction *. Wiley-Interscience, N. Y.. $916 \mathrm{p}$.

TAPPI. 1970. - "Standard Methods *. Technical Association of the Pulp and Paper Institute, N, Y., U. S. A., 393 p.

Van Coillie (R). Dumouchel (A.), Hayeur (G.) Lamothe (P.). 1983. - Evaluation et prévision écologique préalable à l'aménagement hydro-électrique d'un réservoir naturel hé-

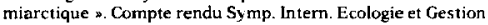
des Réservoirs, Québec. Revue canadienne Ressources Eau., 8: 60-82.

Van Coillie (R.), Visser (S.A.) Couture (P.). 1981. _ * Utilisation de bioessais avec les algues pour l'étude des répercussions lièes à la mise en eau des réservoirs ". Annls Limmol.. $17: 79.91$.

Visser (S.A.), Campbell (P.G.C.) Couture (P.). 1977. - * Impact du flottage du bois sur les eaux du lac Talbot: évaluation à l'aide de tests biologiques". INRS-Eau, Université du Québec, Rapport scientifique $n^{\circ} 77.37 \mathrm{p}$. 